\title{
El pensamiento algebraico, algo más que letras. Una mirada desde la perspectiva semiótica cultural' ${ }^{1}$
}

\author{
Algebraic thinking, more than letters. A look from \\ the perspective of cultural semiotics
}

Pensamento algébrico, mais do que letras. Um olhar a partir da perspectiva da semiótica culturais

Recibido: mayo de 2013

Aprobado: agosto de 2013
John Gómez Triana

\section{Resumen}

En esta comunicación se presentan los resultados obtenidos al implementar una de las Tareas diseñadas para el proyecto de investigación presentado como tesis de maestría de Gómez (2013). Se muestra el análisis realizado a la actividad matemática de los estudiantes foco de la investigación utilizando como marco de referencia el enfoque semiótico-cultural propuesto por Radford $(2008,2009)$ sobre el pensamiento algebraico. El objetivo es socializar una evidencia de la movilización de algunos medios semióticos de objetivación por parte de un grupo de estudiantes de décimo grado de la educación escolar colombiana al resolver una Tarea sobre generalización de patrones en secuencias figurales. Además se utilizan dichos medios semióticos de objetivación para caracterizar las formas de pensamiento algebraico manifestadas por los estudiantes.

Palabras clave: Matemáticas escolares; álgebra; relaciones; pensamiento algebraico; aprendizaje; procesos cognitivos; generalización; generalización de patrones; medios semióticos de objetivación.

\section{Abstract}

In this paper we present the results obtained by implementing one of the tasks designed for the research project presented as Gomez MA thesis (2013). Analysis is shown to the students' mathematical activity focus of research using as a framework the semiotic-cultural approach proposed by Radford $(2008,2009)$ on algebraic thinking. The goal is to socialize evidence of mobilization of some semiotic means of objectification by a group of tenth graders Colombian school education to resolve a generalization Task figural patterns in sequences. Besides using such semiotic means of objectification to characterize the forms of algebraic thinking expressed by the students.

Keywords: school mathematics; algebra, relationships, algebraic thinking, learning, cognitive processes generalization generalization of patterns; semiotic means of objectification. 


\section{Resumo}

Neste artigo, apresentamos os resultados obtidos através da implementação de uma das tarefas projetadas para o projeto de pesquisa apresentado como tese Gomez MA (2013). A análise é mostrado a matemática foco da pesquisa utilizando-se como um quadro de uma abordagem semiótico-cultural proposto por Radford $(2008,2009)$ sobre o pensamento algébrico atividade dos alunos. O objetivo é socializar evidência de mobilização de alguns meios semióticos de objetificação por um grupo de alunos do ensino colombiano décimo para resolver uma generalização de tarefas padrões figurativos em seqüências. Além de usar esses meios semióticos de objetificação para caracterizar as formas de pensamento algébrico manifestado pelos alunos.

Palavras-chave: matemática escolar; álgebra, os relacionamentos, o pensamento algébrico, aprendizagem, processos cognitivos generalização generalização de padrões; meios semióticos de objetificação.

\section{Presentación del problema}

Uno de los intereses actuales en las investigaciones en educación matemática tiene que ver con el estudio de la influencia del contexto sociocultural, de los estudiantes, en el aprendizaje de las matemáticas. Un ejemplo de este interés lo constituyen los trabajos investigativos enmarcados en la perspectiva semiótica cultural que tiene como máximo representante al profesor Luis Radford ${ }^{3}$. En esta perspectiva se tiene como propósito, entre otras cosas, estudiar los recursos semióticos que movilizan los estudiantes para expresar su pensamiento matemático. Estos recursos, caracterizados como medios semióticos de objetivación, tienen un carácter Vygotskiano, en tanto se reconoce que los signos lingüísticos y no lingüísticos son usados por los estudiantes con el propósito de hacer visible y comunicar su pensamiento, en otras palabras, son usados para objetivar el conocimiento.

La presente ponencia se enmarca en la perspectiva semiótica cultural de la educación matemática y tiene como propósito mostrar una evidencia de cómo los estudiantes manifiestan su pensamiento algebraico. Es decir, se pretende, en primer lugar, evidenciar la necesidad de reconocer que las formas de pensamiento algebraico se pueden visualizar a través del cuerpo, el movimiento y de la actividad perceptual. En segundo lugar, se quiere llamar la atención sobre la importancia de identificar procesos en la actividad matemática de los estudiantes que permiten caracterizar las formas de pensamiento algebraico. Estos dos aspectos, los elementos que son movilizados por los estudiantes y los procesos desarrollados por ellos, al parecer están siendo desestimados en el aula de clase de matemáticas y es por esta razón que el desarrollo de la presente comunicación quiere contribuir a poner de manifiesto que el desestimar los aspectos ya mencionados podría ser un obstáculo en la comprensión del aprendizaje de las matemáticas, más específicamente en la comprensión de elementos didácticos vinculados con el pensamiento algebraico.

Los anteriores planteamientos están motivados por el hecho de que, desde una perspectiva semiótica cultural, el álgebra trata de hacer frente a la indeterminación de una manera analítica, pero en lugar de conceder al simbolismo alfanumérico estándar del álgebra el derecho exclusivo de designar y expresar la indeterminación, se debe considerar como sólo una de las varias formas semióticas equipadas para lograrlo, ya que los signos pueden también ser algo muy diferente, palabra o gestos, por ejemplo son signos, semióticamente hablando, algebraicos tan auténticos como las letras Radford (2010).

3 Profesor de la Université Laurentienne, Ontario-Canadá 


\section{Marco de referencia conceptual}

La presente ponencia tiene su marco de referencia conceptual basado en la concepción de álgebra y pensamiento algebraico desde el enfoque semiótico-cultural y toma los elementos teóricos de la Teoría Cultural de la Objetivación (TCO) desarrollada por Radford (2006). En esta teoría se desarrolla una concepción de pensamiento como practica social, como una reflexión mediatizada del mundo. Se profundiza la idea de signo desde la perspectiva de Vygotski. Se sostiene que los recursos semióticos son instrumentos constitutivos del pensamiento introduciendo la idea de Medios Semióticos de Objetivación (artefactos, gestos, símbolos, palabras) considerada como recursos semióticos. Los medios semióticos de objetivación no son únicamente herramientas por medio de las cuales manipulamos el mundo, sino mediadores de nuestros actos intencionales, portadores de una conciencia histórica construida a partir de la actividad cognitiva de las generaciones precedentes Radford $(2008,2010)$.

Además de los elementos teóricos expuestos en el párrafo anterior, (Radford, 2010) propone,

en el contexto de la generalización de patrones, una tipología de formas de pensamiento algebraico que se expone a continuación:

- El Pensamiento Algebraico Factual. Aquí la indeterminación queda implícita en palabras y gestos y el ritmo constituye la sustancia de la semiótica en los estudiantes en un proceso llamado fórmulas en acción.

- El Pensamiento Algebraico Contextual. Aquí la indeterminación se convierte en un objeto explícito del discurso. Los gestos y ritmos son reemplazados por deícticos lingüísticos, adverbios, etc.

- El Pensamiento Algebraico Simbólico. Aquí las fórmulas en lugar de ser un dispositivo de resumen de cálculos aparecen como narraciones vividas; son íconos que ofrecen una especie de descripción espacial de la figura y acciones que se llevarán a cabo.

El concepto de medio semiótico de objetivación y la tipología de las formas de pensamiento algebraico constituyen las categorías de análisis de los datos presentados en esta ponencia.

\section{Metodología y análisis de datos}

Los datos presentados en este escrito son tomados del proyecto de investigación presentado como tesis de maestría de Gómez (2013) en el que se realizó un estudio de los medios semióticos de objetivación y procesos de objetivación desarrollados por un grupo de estudiantes de grado décimo de un colegio público de la ciudad de Bogotá-Colombia.

Atendiendo al propósito de la presente comunicación, se presentarán los resultados obtenidos del análisis de la actividad matemática de un grupo de tres estudiantes de grado décimo que fue tomado como grupo focal en la tesis ya mencionada. La recolección de información se realizó en el instrumento presentado en la Figura 1 y por medio de grabaciones de vídeo del proceso de solución de los estudiantes. Los resultados se obtuvieron del análisis multimodal ${ }^{4}$ del proceso de solución desarrollado por el grupo al enfrentarse a una de las Tareas diseñadas para la investigación (Ver Figura $1)$.

Los resultados que se presentarán aquí se centran exclusivamente al punto tres de la Tarea (Escribir un mensaje a un estudiante de otra clase, con todos los detalles, en el que se indique la manera de averiguar el número de cuadros de cualquier figura) ya que en el proceso realizado por los estudiantes en este punto se movilizaron una serie de medios semióticos de objetivación que dan cuenta de una de las formas de pensamiento algebraico propuestas por Radford (2010), el pensamiento algebraico contextual. La siguiente transcripción corresponde a la explicación de uno de los estudiantes del grupo sobre la manera de hallar el número de cuadros de

4 En este tipo de análisis, se considera que lo gestuado, lo hablado y lo escrito por los estudiantes se analiza de manera conjunta, es decir, interesa centrar la mirada en la relación de los distintos sistemas semióticos activados en la actividad matemática. 
cualquier figura de la secuencia presentada en la Figura 1.

Figura 1

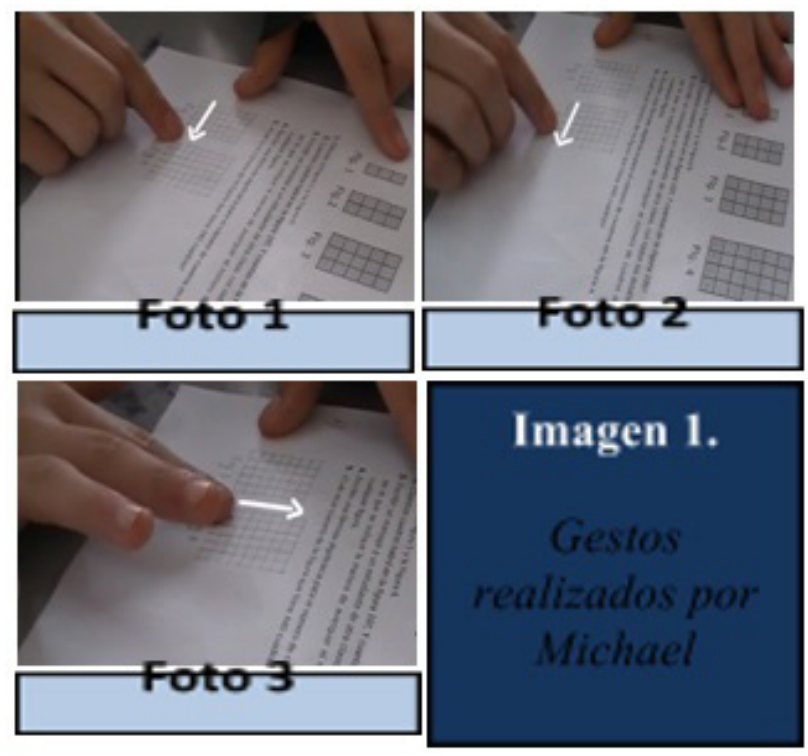

Fuente: Elaboración propia

En la transcripción se puede observar cómo Michael explica paso a paso la forma de hallar el número de cuadrados de cualquier figura tomando como ejemplo la figura 4 de la secuencia. En el discurso de Michael se evidencia la preferencia del grupo por realizar dos cálculos independientes: "Primero coger la figura que necesita y sumarle 2 y ese resultado multiplicarlo por la figura que está". En la Foto 1 se observa un movimiento realizado por Michael indicando que inicialmente se debe calcular el número de cuadros verticales, en la Foto 2 el movimiento es para indicar que el número de cuadros horizontales coincide con el número de la figura y en la Foto 3 Michael realiza un movimiento sobre toda la figura explicando que al multiplicar los dos números obtenidos se tendrá como resultado el número total de cuadros de la figura. En este episodio de la actividad matemática de los estudiantes la indeterminación está presente en frases como "la figura que necesita" y "la figura que está".

Como ya se ha mencionado, la explicación ofrecida por Michael está referida al punto 3 de la Tarea mostrada en la Figura 1(escribir un mensaje a un estudiante de otra clase en el que se indique la manera de averiguar el número de círculos de cualquier figura), la actividad matemática de los estudiantes evidenció Pensamiento Algebraico Contextual ya que la indeterminación se convirtió en objeto explicito del discurso en las frases "la figura que necesita” y "la figura que está".

Por otro lado, el grupo utiliza la estructura espacial de las figuras para plantear dos expresiones algebraicas, una para calcular el número de cuadros verticales y la otra para calcular el número de cuadros de toda la figura. Los estudiantes explican cómo hallar el número de círculos de una figura cualquiera, en el punto 2 de la Tarea construyen

Figura 2

Profesor: Michael le va explicar a Cristian cómo hallar el número de cuadritos de cualquier figura

Michael: Primero coger la figura que necesita y sumarle 2 y ese resultado multiplicarlo por la figura que está, entonces acá vo lo hice. Cuatro le sume dos y me dio seis, que son los numeros de cuadros que hay hacia arriba (Ver Foto 1) y ese seis lo multiplique por cuatro que es el mismo numero de la figura y me dio el total de los cuadritos, porque hay uno, dos, tres, cuatro, cinco, seis [el estudiante sube el tono de la voz cuando dice "seis"] hacia arriba por cuatro (Ver Foto 2) da veinticuatro en total (Ver Foto 3). Toca al número de la figura sumarle dos y el total multiplicarlo por el número de la figura y hay da el total de los cubitos.

Fuente: Elaboración propia 
la fórmula algebraica siendo, representa la figura y la letra se utiliza para representar el número de cuadros de cualquier figura. El significado que tienen, para los estudiantes, estos símbolos algebraicos puede ser una evidencia de Pensamiento Algebraico Simbólico ya que su utilización sugiere que aparecen como narraciones vividas y son íconos que ofrecen una especie de descripción espacial de la figura y acciones que se llevarán a cabo (Radford, 2010)

\section{Conclusiones}

En el desarrollo de esta Tarea el grupo se fijó en la configuración espacial de las figuras, utilizando dicha configuración para determinar la relación existente entre el número de cuadros de la altura del rectángulo (cuadros verticales) y el número de cuadros de la base del rectángulo (cuadros horizontales). Identificaron que el número de cuadros verticales es igual al número de cuadros horizontales más uno y que el número de cuadros horizontales coincide con el número de la posición de la figura. Radford (2012) señala que la vinculación entre las estructuras espaciales y numéricas es un aspecto importante en el proceso de generalización. Tal importancia radica en la transformación cultural que se produce en la manera de ver la secuencia, una transformación que puede llamarse domesticación del ojo.

En lo que tiene que ver con los medios semióticos de objetivación, en el segmento de actividad matemática presentado en este escrito se muestra una evidencia de la movilización de dos medios semióticos de objetivación. El primero se denomina Medio semiótico de objetivación movimiento y consiste en un gesto utilizado por los estudiantes para establecer relaciones geométrico-espaciales en una figura, relaciones que les permitió identificar el patrón de construcción de la secuencia figural. El segundo se denomina Medio semiótico de objetivación "la figura que se necesite" y es un recurso semiótico que fue usado por los estudiantes para designar la variable dependiente de la secuencia, es decir, les permitía expresar la figura sin utilizar símbolos alfanuméricos algebraicos. Este medio semiótico de objetivación estuvo acompañado por la frase "la figura que está", frase que fue utilizada por los estudiantes para designar la variable independiente.

En síntesis, en la presente comunicación se muestra una evidencia de que el pensamiento algebraico se manifiesta a través de medios semióticos de objetivación diferentes al simbolismo estándar del algebra (las letras). Además es importante señalar que estos medios semióticos de objetivación fueron utilizados por los estudiantes para proporcionar una solución satisfactoria a la Tarea presentada, lo que implica que la no manipulación de los símbolos algebraicos no constituye un obstáculo para desarrollar dicha Tarea.

\section{Referencias}

Gómez, J. (2013). La generalización de patrones de secuencias figurales y numéricas: Un estudio de los medios semióticos de objetivación $y$ procesos de objetivación en estudiantes de grado décimo. Tesis de maestría no publicada. Universidad Pedagógica Nacional. Bogotá-Colombia

Radford, L. (2006). Elementos de una teoría cultural de la objetivación. Revista Latinoamericana de Investigación en Matemática Educativa, número especial sobre semiótica, cultura y pensamiento matemático (editores invitados: L. Radford y B. D’Amore), pp. 267-299.

Radford, L. (2008). Iconicity and contraction: a semiotic investigation of forms of algebraic generalizations of patterns in different contexts. ZDM Mathematics Education, 40, 83-96.

Radford, L. (2010). Algebraic thinking from a cultural semiotic perspective. Research in Mathematics Education, 12(1), 1-19.

Radford, L. (2012). The eye as a theoretician: Seeing structures in generalizing activities, For the Learning of Mathematics, 30(2), 2-7. 